\title{
Visualization of diffusion and convection heat transport in a square cavity with natural convection
}

\author{
Moghtada Mobedi a ${ }^{\mathrm{a}}$ Ünver Özkol ${ }^{\mathrm{a}}$, Bengt Sunden ${ }^{\mathrm{b}, *}$ \\ a Department of Mechanical Engineering, Izmir Institute of Technology, 35430 Urla, Izmir, Turkey \\ ${ }^{\mathrm{b}}$ Division of Heat Transfer, Lund University, P.O. Box 118, SE-22100 Lund, Sweden
}

\section{A R T I C L E I N F O}

\section{Article history:}

Received 28 November 2008

Accepted 18 September 2009

Available online 29 October 2009

\section{Keywords:}

Convective flow

Vorticity

Heatlines

Diffusion

Convection

\begin{abstract}
A B S T R A C T
In this study, the total heatfunction equation which includes diffusion and convection transport is divided into the corresponding heatfunction equations. The superposition rule is used to obtain the mathematical definitions of diffusion and convection heatfunctions and corresponding boundary conditions. It is observed that the separate visualization of diffusion and convection heatlines provides significant information on understanding of the mechanism of heat transfer in a convective flow. The direction of the diffusion and convection heat transport as well as the strength of convection compared to the conduction in entire or in a portion of a domain can be visualized. The diffusion heatlines demonstrate a potential flow like behavior while convective heat flow rotates due to the source term of the convection heatfunction equation, similar to the rotation of fluid flow generated by fluid flow vorticity. The similarity between the streamfunction and the total heatfunction yields a concept of heat flow vorticity, $\Omega_{t}$. The obtained results show that the maximum absolute value of the convection heatfunction may be an appropriate parameter for determination of the convection strength. The diffusion and convection heatfunction equations for natural convection in a differentially heated square cavity for four different length of the heated surface on the right vertical wall as $s_{\mathrm{p}}=L / 4, L / 2,3 L / 4$ and $L$ and a fixed length of the cooled surface on the right vertical wall as $L / 4$ are obtained and corresponding heatlines are drawn. The values of the conduction heatfunction are positive while the sign of convection heatfunction values is negative for the studied cases. Based on the distribution of total heatlines, two regions are detected in the cavity, an active region with the positive values of heatlines signifying dominant conduction heat transfer and a passive region with the negative heatfunction values in where convection heat flow is dominant and heat only rotates in a closed contour pattern. The variations of average Nusselt number, average of heat flow vorticity, maximum absolute values of convection heatfunction and streamfunction at different Rayleigh numbers and lengths of the heated surface are presented.
\end{abstract}

(c) 2009 Elsevier Ltd. All rights reserved.

\section{Introduction}

In many computational heat transfer studies, isotherms and streamlines are used to explain the mechanism of heat transfer in a domain. It is not easy to understand the direction and strength of heat transfer in a convective flow since heat flux lines are not perpendicular to isotherms. Hence, heatline technique was developed to visualize the direction and intensity of heat flux in a convective domain. The heatlines provide corridors in where heat is transferred from a hot to a cold region by convection and/or conduction.

The heatfunction concept was introduced by Kimura and Bejan [1] for natural convection in a differentially heated square enclosure whose top and bottom boundaries were adiabatic. Then, the

\footnotetext{
* Corresponding author. Tel.: +46 46222 8605; fax: +46 462224717.

E-mail address: bengt.sunden@energy.lth.se (B. Sunden).
}

massline concept was proposed for convective mass transfer problems [2]. Morega and Bejan [3] and Nield and Bejan [4] visualized heatlines in forced convection laminar boundary layers and in porous media, respectively. A review study was reported by Costa [5] to summarize the application of heatline visualization technique in different heat and mass transfer problems. He showed that heatlines can be applied to the boundary layer problem, turbulent flow, reacting flow, fluid-saturated porous media for both isotropic and anisotropic media. Costa [6] also unified the streamline, heatline and massline methods in order to provide a common procedure. Mukhopadhyay et al. [7] unified the heat and massline methods for a nonreacting jets of hot air in cold air, nonreacting methane and hydrogen jets in air, as well as non-premixed reacting jets of methane and air. Zhao et al. [8] performed a study on application of the streamline, heatline and massline methods for conjugate heat and mass transfer. Dalal and Das [9] visualized heat transport by the heatline technique for a complicated enclosure. Mobedi 


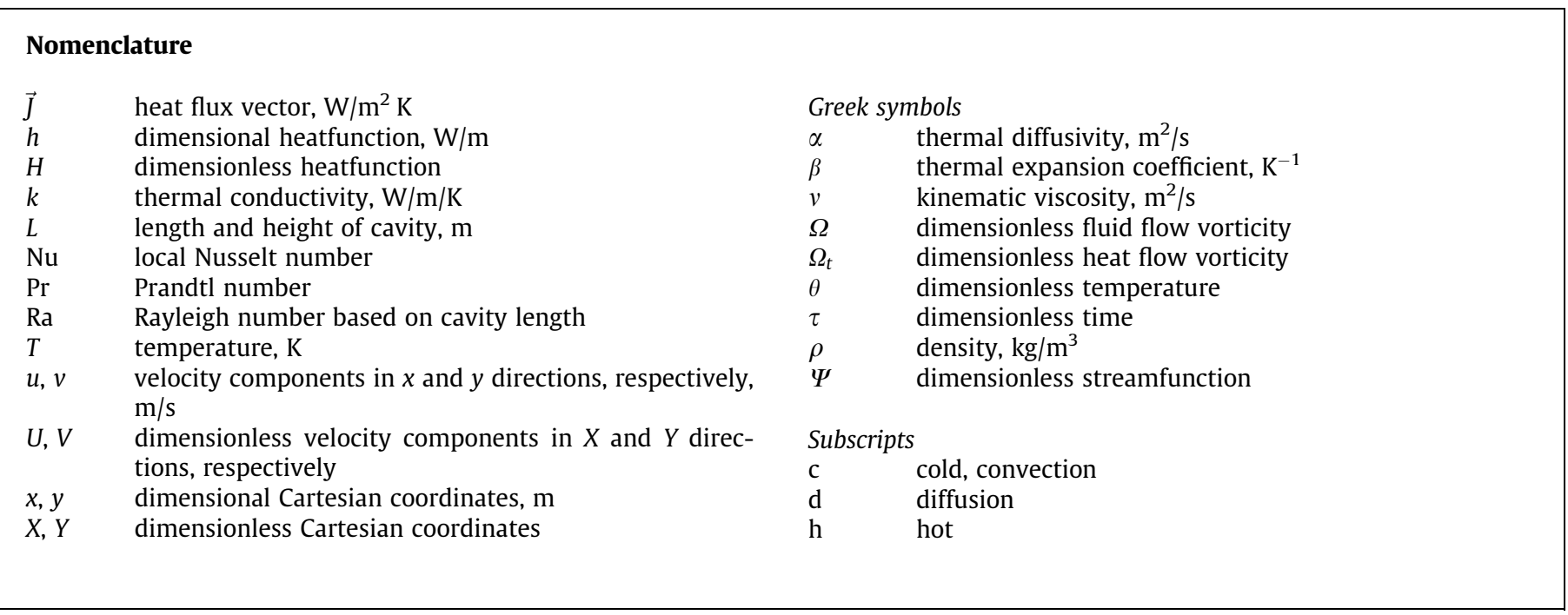

et al. [10,11] used the heatline technique to observe heat transport in the entire domain of a square cavity with thick horizontal walls. Recently, the heatline and streamline formulations were employed by Basak and Roy [12] to demonstrate the heat flow for differentially and distributed heating walls within cavities. Deng and Tang [13] used heatlines to show the structure of heat flow for a twodimensional, steady state and laminar natural convection in a rectangular enclosure with discrete heat sources on walls. Deng et al. [14] also used streamfunction, heatfunction and massfunction definitions to demonstrate the path of the fluid, heat and contaminant transport in a two-dimensional ventilated enclosure with mixed convection.

The problem of natural convection heat transfer through a cavity with partially heated vertical walls has been also investigated by researchers. For the rectangular cavities with adiabatic ceiling and floor, the following studies were reported in the literature. Poulikakos [15] investigated natural convection in a cavity with a single vertical wall with warm and cold regions. The effect of heater and cooler locations on natural convection in square cavities is studied by Yücel and Türkoglu [16]. Nithyadevi et al. [17] worked on natural convection heat transfer in the cavities with partially active sides for different aspect ratios. Deng [18] investigated the size and arrangement effects of the heat sources and sinks on the fluid flow and heat transfer in a square cavity. Valencia and Frederick [19] studies heat transfer in square cavities with partially active vertical walls. The problem of natural convection in shallow enclosures with differentially heated end walls was studied by Paolucci and Chenoweth [20]. Ho and Chang [21] investigated natural convection heat transfer in a vertical rectangular enclosure with two-dimensional discrete heating.

The aim of the present study is to obtain separate heatfunction equations for the diffusion and convection modes of heat transport in a convective flow. The total heatfunction equation which includes both diffusion and convection modes of heat transport demonstrates the path and intensity of the total heat transfer. The separation of the total heatfunction into diffusion and convection heatfunctions helps in distinguishing the path and intensity of each mode of heat transfer in a domain. Hence, the effect of each mode of heat transfer in a convective flow can be assessed. The present study mathematically and physically explains the meaning of the source term on the right side of total heatfunction equation and the negative values of total heatlines observed in studies employed heatline visualization technique. The superposition rule is used to separate the total heatfunction equations into the diffusion and convection heatfunction equations. The diffusion, convection and total heatlines are plotted for the partially heated cavities with different Rayleigh numbers and four lengths of heat source.

\section{Physical domain}

The square cavity for which the diffusion and convection heatlines are plotted is shown in Fig. 1. Heat is transferred from the left to the right wall by natural convection and the top and bottom walls are adiabatic. The symbol $S_{\mathrm{p}}$ shows the length of the heated surface which always has one edge on the left top corner. Gravity acts in the vertical direction but the radiation mode of heat transfer is neglected. The study is performed for four different lengths of the heat source as $s_{\mathrm{p}}=L / 4, L / 2,3 L / 4$ and $L$ while the length of cooled surface is kept the same at $L / 4$. The heated and the cooled surfaces are isothermal, though the remaining walls are adiabatic. The numerical results are obtained for air with $\operatorname{Pr}=0.7$ and $10^{2}<\mathrm{Ra}<10^{6}$

\section{Mathematical formulation}

\subsection{Determination of streamfunction and temperature fields}

The governing equations for the problem are continuity, momentum and energy equations. The unsteady forms of the governing equations are solved to obtain the steady state results. The vorticity-streamfunction approach is used to predict the velocity field. By employing the dimensionless vorticity and streamfunction parameters, the dimensionless forms of the equations to be solved can be written as:

$$
\frac{\partial \Omega}{\partial \tau}+\frac{\partial U \Omega}{\partial X}+\frac{\partial V \Omega}{\partial Y}=\operatorname{Pr}\left(\frac{\partial^{2} \Omega}{\partial X^{2}}+\frac{\partial^{2} \Omega}{\partial Y^{2}}\right)+\operatorname{Ra} \cdot \operatorname{Pr} \cdot \frac{\partial \theta}{\partial X}
$$

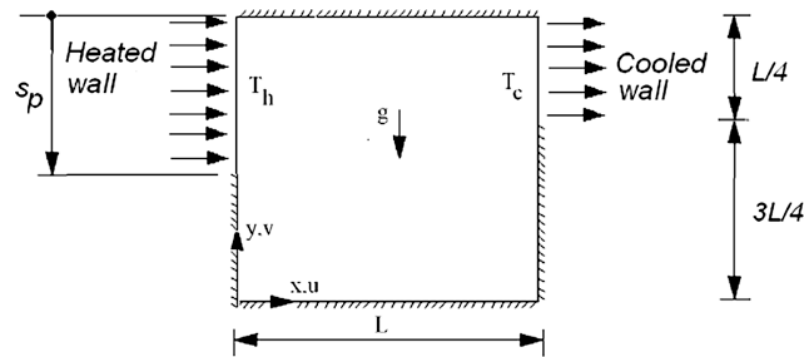

Fig. 1. Schematic view of the studied cavity. 
$\frac{\partial^{2} \Psi}{\partial X^{2}}+\frac{\partial^{2} \Psi}{\partial Y^{2}}=-\Omega$

$\frac{\partial \theta}{\partial \tau}+\frac{\partial U \theta}{\partial X}+\frac{\partial V \theta}{\partial Y}=\frac{\partial^{2} \theta}{\partial X^{2}}+\frac{\partial^{2} \theta}{\partial Y^{2}}$

where $U, V, \theta, \tau, X$ and $Y$ are dimensionless parameters defined as:

$X=\frac{x}{L}, \quad Y=\frac{y}{L}, \quad U=\frac{u L}{\alpha}, \quad V=\frac{v L}{\alpha}, \quad \theta=\frac{\left(T-T_{\mathrm{c}}\right)}{\left(T_{\mathrm{h}}-T_{\mathrm{c}}\right)}, \quad \tau=\frac{\alpha t}{L^{2}}$

The dimensionless vorticity, streamfunction, Rayleigh and Prandtl numbers are defined as:

$\Omega=\frac{\partial V}{\partial X}-\frac{\partial U}{\partial Y}$

$U=\frac{\partial \Psi}{\partial Y}, \quad V=-\frac{\partial \Psi}{\partial X}$

$\mathrm{Ra}=\frac{g \beta\left(T_{\mathrm{h}}-T_{\mathrm{c}}\right) L^{3}}{v \alpha}, \quad \operatorname{Pr}=\frac{v}{\alpha}$

The boundary conditions for the domain shown, in Fig. 1, can be written as follows:

$Y=0, \quad Y=1 ; \quad \Omega=-\frac{\partial U}{\partial Y}, \quad \Psi=0, \quad \frac{\partial \theta}{\partial Y}=0$

$X=0 ; \quad \Omega=\frac{\partial V}{\partial X}, \quad \Psi=0$

for $0<Y<1-S_{\mathrm{p}} ; \quad \frac{\partial \theta}{\partial X}=0$

for $1-S_{\mathrm{p}}<Y<1 \quad \theta(0, Y, \tau)=1$

$X=1 ; \quad \Omega=\frac{\partial V}{\partial X}, \quad \Psi=0$

for $0<Y<0.75 \quad \frac{\partial \theta}{\partial X}=0$

for $0.75<Y<1 \quad \theta(1, Y, \tau)=0$

where $S_{\mathrm{p}}$ is a dimensionless parameter and it is defined as $S_{\mathrm{p}}=S_{\mathrm{p}} / L$. Based on the definition of the streamfunction (Eq. (6)) and considering the defined boundary conditions, the positive sign of $\Psi$ denotes anti-clockwise circulation, and the clockwise circulation is represented by the negative sign of $\Psi$. The following initial values for the dimensionless temperature, vorticity and streamfunction are used:

$U=V=\Omega=\Psi=\theta=0$

\subsection{Definitions of dimensional and dimensionless total heatfunction equation}

For a two-dimensional steady and incompressible convective flow without heat generation, the components of the total heat flux vector, containing the diffusion and convection transport, in $x$ and $y$ directions can be written as:

$J_{x}=\rho c_{P} u\left(T-T_{\mathrm{c}}\right)-k \frac{\partial T}{\partial x}, \quad J_{y}=\rho c_{P} v\left(T-T_{\mathrm{c}}\right)-k \frac{\partial T}{\partial y}$

where $T_{\mathrm{c}}$ is the reference temperature. The total heat flux vector, $\vec{J}$, is the vectorial sum of the two components;

$\vec{J}=J_{x} \vec{i}+J_{y} \vec{j}$
The vectors $\vec{i}$ and $\vec{j}$ represent Cartesian unit vectors. The application of the energy conservation law on a finite element in a flow field and considering the total heat flux definition (Eq. (17)) yield the energy equation for an incompressible steady convective flow:

$\frac{\partial J_{x}}{\partial x}+\frac{\partial J_{y}}{\partial y}=\rho c_{p}\left(\frac{\partial u T}{\partial x}+\frac{\partial v T}{\partial y}\right)-k\left(\frac{\partial^{2} T}{\partial x^{2}}+\frac{\partial^{2} T}{\partial y^{2}}\right)=0$

By assuming $h$ as a continuous scalar function, the dimensional heatfunction can be defined in a differential form [1]:

$-\frac{\partial h}{\partial x}=J_{y} ; \quad \frac{\partial h}{\partial y}=J_{x}$

By substituting of Eq. (19) into Eq. (16), taking derivatives with respect to $y$ and $x$, and subtracting the resulting equations from each other, an elliptic partial differential equation for the total heatfunction can be obtained:

$\frac{\partial^{2} h}{\partial x^{2}}+\frac{\partial^{2} h}{\partial y^{2}}=\rho c_{\mathrm{P}}\left(\frac{\partial u T}{\partial y}-\frac{\partial v T}{\partial x}\right)$

The convection term which appears on the right side of Eq. (20) acts as a source term. It should be mentioned that the defined heatfunction, $h$, includes both the diffusion and convection modes of heat transfer. The solution of Eq. (20) yields the values of the total heatfunction for the all nodes of a computational domain and contour plots of the heatfunction values provides heatline patterns. By employing the dimensionless parameters presented in Eq. (4), the dimensionless total heatfunction can be written as:

$-\frac{\partial H}{\partial X}=V \theta-\frac{\partial \theta}{\partial Y} ; \quad \frac{\partial H}{\partial Y}=U \theta-\frac{\partial \theta}{\partial X}$

where $H$ is the dimensionless total heatfunction as:

$H=\frac{h}{\left(T_{\mathrm{h}}-T_{\mathrm{c}}\right) k}$

By performing the mathematical manipulations similar to the procedure for obtaining Eq. (20) from Eq. (19), the following dimensionless total heatfunction equation can be obtained from Eq. (21):

$\frac{\partial^{2} H}{\partial X^{2}}+\frac{\partial^{2} H}{\partial Y^{2}}=\frac{\partial U \theta}{\partial Y}-\frac{\partial V \theta}{\partial X}$

The boundary conditions for dimensionless total heatfunction (Eq. (23)) are obtained from the integration of Eq. (21) along the considered boundary. For instance, the following equation can be used to determine the values of heatfunction at the right wall of the cavity [6]:

For $X=0 ; \quad 0<Y \leq 1 \quad H(0, Y)=H(0,0)-\left.\int_{0}^{Y} \frac{\partial \theta}{\partial X}\right|_{X=0} d Y$

The derivative of the dimensionless temperature at a wall with respect to its normal direction is defined as local Nusselt number, hence, Eq. (24) can also be written in the following form:

For $X=0 ; \quad 0<Y \leq 1 \quad H(0, Y)=H(0,0)-\left.\int_{0}^{Y} \mathrm{Nu}(Y)\right|_{X=0} d Y$

\subsection{Application of superposition}

The Eq. (23) is linear, since the values of $U, V$ and $\theta$ are known from solution of the continuity, momentum and energy equations and therefore the superposition method can be used. The sum of two heatfunctions is also a heatfunction and describes a heat flux 
field. Hence, the total heatfunction can be separated into two heatfunctions as $H_{\mathrm{d}}$ and $H_{\mathrm{c}}$, as:

$H=H_{\mathrm{d}}+H_{\mathrm{c}}$

$H_{\mathrm{d}}$ and $H_{\mathrm{c}}$ represent the diffusion and convection heatfunctions, respectively, and can be defined as:

$\frac{\partial H_{\mathrm{d}}}{\partial X}=\frac{\partial \theta}{\partial Y} ; \quad \frac{\partial H_{\mathrm{d}}}{\partial Y}=-\frac{\partial \theta}{\partial X}$

and

$-\frac{\partial H_{\mathrm{c}}}{\partial X}=V \theta ; \quad \frac{\partial H_{\mathrm{c}}}{\partial Y}=U \theta$

Mathematical manipulation can be carried out on Eqs. (27) and (28), and separate heatfunction equations for the diffusion and convection modes of heat transfer can be found:

$\frac{\partial^{2} H_{\mathrm{d}}}{\partial X^{2}}+\frac{\partial^{2} H_{\mathrm{d}}}{\partial Y^{2}}=0$

$\frac{\partial^{2} H_{c}}{\partial X^{2}}+\frac{\partial^{2} H_{c}}{\partial Y^{2}}=\frac{\partial U \theta}{\partial Y}-\frac{\partial V \theta}{\partial X}$

There is no source term in the diffusion heatfunction equation (Eq. (29)) and the values of $H_{\mathrm{d}}$ are influenced only from the boundary conditions. The boundary conditions for the diffusion heatfunction equation can be written by the same method as described for the total heatfunction (Eq. (24)). The values of the convective heatfunction on the walls are zero, hence $H_{c}=0$ for the all walls. In the present study, the value of the diffusion heatfunction at the origin is taken as zero, $H_{\mathrm{d}}(0,0)=0$.

\subsection{Similarity between fluid and heat flows}

The term on the right hand side of the streamfunction equation (Eq. (2)) represents vorticity, $\Omega$, which can be related to the pointwise rotation rate of a fluid element and its sign refers to the direction of pointwise rotation as shown in Fig. 2(a). Similar to an isothermal fluid flow, the right term of Eq. (23) represents the pointwise rotation rate of an element in the heat flow field (Fig. 2(b)). This pointwise heat flow rotation can be called as heat flow vorticity, $\Omega_{t}$.

$\Omega_{t}=\frac{\partial V \theta}{\partial X}-\frac{\partial U \theta}{\partial Y}$

The main reason for a rotational heat flow is the gradients of the convective heat flux components, $U \theta$ and $V \theta$. For an isothermal flow the Eq. (31) become as:
$\Omega_{t}=\theta\left(\frac{\partial V}{\partial X}-\frac{\partial U}{\partial Y}\right)$

However, for a flow field with uniform $U$ and $V$ velocity components but with the presence of temperature gradient, the Eq. (31) takes the following form:

$\Omega_{t}=V \frac{\partial \theta}{\partial X}-U \frac{\partial \theta}{\partial Y}$

For an isothermal flow, the fluid flow vorticity is the reason for the convective heat flow rotation; however for a uniform velocity field, the temperature gradient causes the rotation of convective heat flow. A requirement for existence of an irrotational heat flow is:

$\Omega_{t}=\frac{\partial U \theta}{\partial Y}-\frac{\partial V \theta}{\partial X}=0$

This requirement changes the heatfunction equation (Eq. (23)) to the following form:

$\frac{\partial^{2} H}{\partial X^{2}}+\frac{\partial^{2} H}{\partial Y^{2}}=0$

An example for an irrotational heat flow is pure heat conduction. For pure conduction heat transfer, the heatlines become identical to the heat flux lines which are well known from heat transfer text books. On the other hand, an irrotational convective heat flow may exist if the convection heat flow satisfies the irrotational heat flow condition (Eq. (34)).

Table 1 demonstrates some of the similarities between heat and fluid flows. As is seen from the table, both $\vec{V}$ and $\vec{J}$ are vectorial quantities referring to the magnitude and direction of fluid and heat transport, respectively. Both $\vec{V}$ and $\vec{J}$ have two components in the $X$ and $Y$ directions in a two-dimensional Cartesian coordinate system. The application of the conservation of mass to a flow field yields the continuity equation and the energy equation is obtained by the application of the conservation of energy to a heat flow field. The fluid or heat flow rate is determined by integration of $\vec{V}$ or $\vec{J}$ over a surface, respectively. A flow field function, as $\psi$ for a fluid flow and $H$ for a heat flow, can be similarly defined. The isolines of these functions provide corridors in which fluid or heat flows. A vectorial quantity called as the fluid flow vorticity, $\vec{\Omega}$, can be defined for a fluid flow field which refers to the magnitude and direction of the pointwise rotation. A similar quantity can be called as the heat flow vorticity, $\vec{\Omega}_{t}$, can also be defined for a heat flow field and it refers to the pointwise rotation of heat. As is seen from Table 1, the partial differential equations which show a relation between the flow field function and the vorticity for both fluid and heat flows are similar.
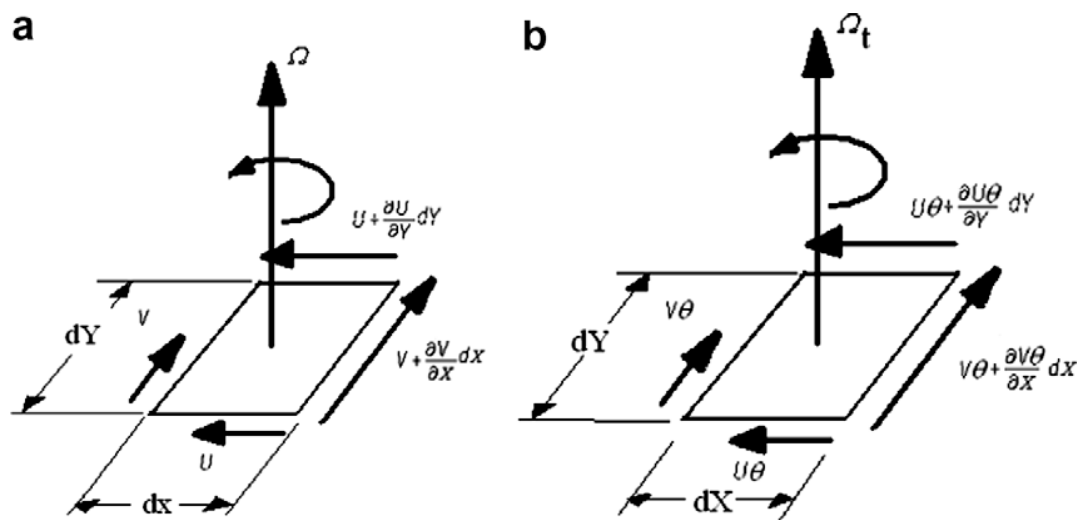

Fig. 2. Flow rotation for an infinitesimal rectangular fluid element, (a) pointwise fluid flow rotation and (b) pointwise heat flow rotation. 
Table 1

Some similarities between heat and fluid flow.

\begin{tabular}{lll}
\hline Parameter & Fluid flow & Heat flow \\
\hline Vectorial flow quantity & $\vec{V}$ & $\vec{J}$ \\
& $\vec{V}=U \vec{i}+V \vec{j}$ & $\vec{J}=J_{x} \vec{i}+J_{y} \vec{j}$ \\
Conservation law & Conservation of & Conservation of \\
& mass & Energy \\
Flow rate & $\frac{\partial U}{\partial X}+\frac{\partial V}{\partial Y}=0$ & $\frac{\partial J_{X}}{\partial X}+\frac{\partial J_{y}}{\partial Y}=0$ \\
Flow field function & $Q=\int_{A} \vec{V} \cdot \vec{n} \cdot d A$ & $Q=\int_{A} \vec{J} \cdot \vec{n} \cdot d A$ \\
Measure of pointwise & $\psi$ & $H$ \\
$\quad$ rotation & $\vec{\Omega}=\frac{\partial V}{\partial X}-\frac{\partial U}{\partial Y}$ & $\Omega_{t}=\frac{\partial V \theta}{\partial X}-\frac{\partial U \theta}{\partial Y}$ \\
Flow field function equation & $\frac{\partial^{2} \psi}{\partial X^{2}}+\frac{\partial^{2} \psi}{\partial Y^{2}}=-\Omega$ & $\frac{\partial^{2} H}{\partial X^{2}}+\frac{\partial^{2} H}{\partial Y^{2}}=-\Omega_{t}$ \\
\hline
\end{tabular}

\section{Solution method}

The set of governing equations is solved by starting from an initial state. The vorticity equation is solved for a time step to compute the vorticity field in the computational domain. Then, the streamfunction equation is solved and the velocity values are obtained from the streamfunction field. At the same time step and by using the new values of velocity, the energy equation is solved and the temperature field is computed. The procedure is continued until the steady state is reached. The steady results of the velocity and temperature fields are used to solve the conduction and convection heatfunction equations. The energy and vorticity equations are solved line by line by employing the Alternating Direction Implicit method (ADI method), whereas the streamfunction and heatfunction equations are solved point by point. The finite difference form of diffusion and convection terms are written based on three points central difference which has a second order accuracy. The outer iteration convergence criterion for the solution of vorticity, energy and streamfunction equations is:

$\frac{\sum\left|\theta^{n+1}-\theta^{n}\right|}{\Delta \tau \sum \theta^{n}} \leq 10^{-3}$

The local and average Nusselt numbers for the cold wall are calculated by the following relations:

$\mathrm{Nu}=-\left.\frac{\partial \theta}{\partial X}\right|_{X=1}$
$\overline{\mathrm{Nu}}=\int_{Y=0}^{Y=1} \mathrm{Nu}(Y) d Y$

The average value of the heat flow vorticity in the cavity is used for the discussion of the results. It is determined by the following equation:

$\left|\bar{\Omega}_{t}\right|=\frac{\iint_{A}\left|\Omega_{1}(X, Y)\right| d A}{\iint_{A} d A}$

Uniform mesh grid sizes are used and the number of nodes in the $\mathrm{X}$ and $\mathrm{Y}$ directions was $200 \times 200$.

\subsection{Validation of numerical results}

In order to validate the employed method and check the written computer code, results are obtained and compared with the benchmark solution of de Vahl Davis [22]. Good agreement between the two solutions is found as seen from Table 2 . The total heatlines drawn by Basak and Roy [12] for natural convection in differentially heated vertical walls with $\mathrm{Ra}=10^{5}$ and $\operatorname{Pr}=0.7$ are plotted and shown in Fig. 3. The heatline patterns of the two studies are al-
Table 2

Comparison of the present numerical results with the solution of de Vahl Davis [22].

\begin{tabular}{cllccl}
\hline \multirow{2}{*}{ Ra } & \multicolumn{2}{l}{ de Vahl Davis } & & \multicolumn{2}{c}{ Present study } \\
\cline { 2 - 3 } \cline { 5 - 6 } & $|\psi|_{\max }$ & $\bar{N} u$ & & $|\psi|_{\max }$ & $\bar{N} u$ \\
\hline $10^{3}$ & - & 1.118 & 1.176 & 1.113 \\
$10^{4}$ & - & 2.243 & 5.077 & 2.237 \\
$10^{5}$ & 9.612 & 4.519 & 9.613 & 4.510 \\
$10^{6}$ & 16.750 & 8.800 & 16.917 & 8.833 \\
\hline
\end{tabular}

most the same proving the accuracy of the present study. It should be mentioned that Basak and Roy solved the continuity, momentum and energy equations with primitive variables approach by the finite element method which is completely different from the present solution method.

\section{Results and discussion}

The isotherms, streamlines, diffusion and convection and total heatlines are plotted for the cavity with partially heated vertical walls for different length of heat source and Rayleigh numbers. Fig. 4 shows the isotherms and streamlines for the cavities with $S_{\mathrm{p}}=0.25$ and $\mathrm{Ra}=10^{2}, 5 \times 10^{3}, 10^{5}$ and $10^{6}$. The figures on the right side are isotherms whereas the left side figures illustrate the streamlines. The isotherms for the $\mathrm{Ra}=10^{2}$ (Fig. 4(a)) exhibits a symmetric distribution with respect to the $X=0.5$ and the magnitudes of the streamfunction are small $\left(|\psi|_{\max }=0.056\right)$ indicating a very slow convective flow and strong conduction heat transfer. Fig. 4(b), illustrating the isotherms and streamlines for the cavity with $\mathrm{Ra}=5 \times 10^{3}$, indicates that the strength of the flow is increased $\left(|\psi|_{\max }=1.866\right)$ with increasing Rayleigh number and as a result the symmetric distribution of isotherms disappears. Moreover, as is seen from Fig. 4(c) and (d), illustrating the isotherms and streamlines for $\mathrm{Ra}=10^{5}$ and $10^{6}$, respectively, the further increase of Rayleigh number enhances the strength of convective heat flow. The maximum value of streamfunction for the cavities with $\mathrm{Ra}=10^{5}$ and $10^{6}$ take values as $|\psi|_{\max }=6.414$ and $|\psi|_{\max }=$ 10.970 , respectively. The distribution of the isotherms is highly influenced from the fluid flow and as a result, the prediction of heat transport path for cavity with strong convection heat transfer becomes troublesome.

Fig. 5 shows the diffusion, convection and total heatlines for the same cavities. The right figures demonstrate the diffusion heatline patterns while the middle figures depict the convection heatlines and the total heatlines are placed on the left column. The diffusion, convection and total heatlines for $\mathrm{Ra}=10^{2}$ are shown in Fig. 5(a). The diffusion heatlines (on the left) demonstrates transfer of heat from the hot to the cold surface by conduction mode. The values of the diffusion heatfunction are positive since heat is transferred in the positive direction of the $X$ axis. The conduction heat transfer is much stronger in the upper region than in the lower area of the cavity. The distribution of convection heatlines (on the middle) is completely different than the diffusion heatlines. The values of the convection heatfunction are zero at the boundaries since velocities are zero at the walls. Similar to the streamfunction, the convection heatfunction takes negative values due to the clockwise rotation of convective heat. The convection heatlines demonstrates the corridors in where heat is circulated due to fluid motion. Lack of diffusion causes no heat transfer from one corridor to an adjacent one. Hence, a circulation of heat only occurs in these corridors. For $\mathrm{Ra}=10^{2}$, the magnitudes of the convection heatfunction are considerably less than those of diffusion heatfunction, $0 \leqslant\left|H_{\mathrm{d}}\right| \leqslant 0.463,0 \leqslant\left|H_{\mathrm{c}}\right| \leqslant 0.0241$, showing the weakness of convection heat transfer. All values of the total heatfunction are positive in the entire region of the cavity and the distribution of the total heatlines is very similar to the distribution of diffusion 

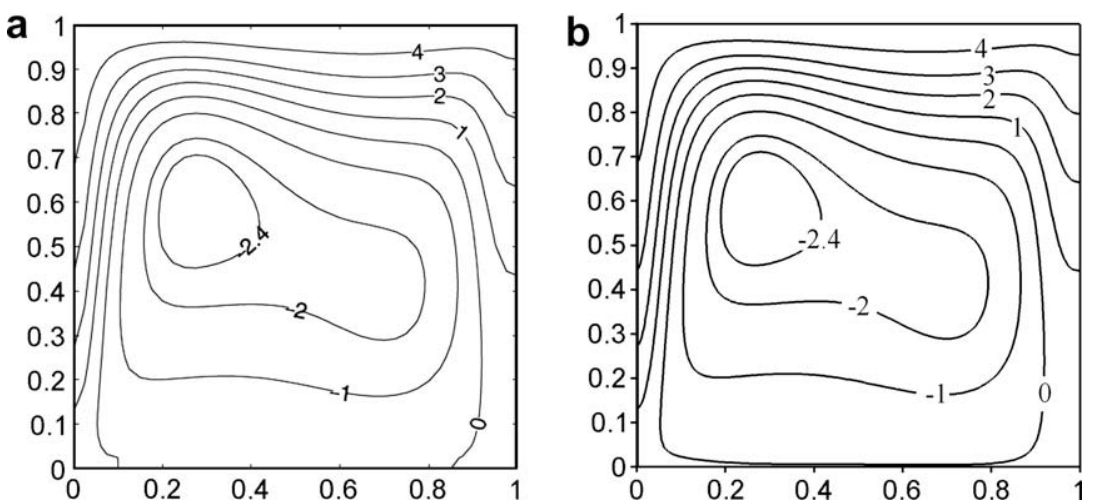

Fig. 3. Total heatlines for a square cavity at $\mathrm{Ra}=10^{5}$ and $\mathrm{Pr}=0.7$. (a) Basak and Roy study [12] and (b) present study.

heatlines due to the strong conduction mode of heat transfer in the cavity.

The diffusion, convection and total heatlines of cavity with $\mathrm{Ra}=5 \times 10^{3}$ are shown in Fig. 5(b). The values of the diffusion heatfunction are positive and they show a similar conduction path to that of $\mathrm{Ra}=10^{2}$. The strength of the convection heat flow is enhanced over 30 -folds $\left(0 \leq\left|H_{c}\right| \leq 0.824\right)$ and the convective heat rotates in the clockwise direction. The distribution of the total heatlines is different from that of cavity with $\mathrm{Ra}=10^{2}$ and a cell, which is colored, appears in the cavity. The values of total heatlines in the cell surrounded by the $H=0$ contour are negative, signifying dominant convection heat transfer in this portion of the cavity. Heat rotates in the cell; however, no diffusion heat transfer occurs. This region of the cavity does not have an active role on the heat transfer from the hot to the cold wall. Fig. 5(c) illustrates three types of heatlines for a partially heated cavity with $\mathrm{Ra}=10^{5}$. The values of the diffusion heatfunction are positive and the increase of Rayleigh number causes the enhancement of the convection mode of heat transfer, $0 \leq\left|H_{c}\right| \leq 2.340$. A clockwise convection cell with negative values of the total heatfunction in the cavity exists. The total heatlines are clustered on the upper region of the cavity and the passive convection cell with negative values of the total heatfunction is enlarged.

The highest convection strength of this study is observed for $\mathrm{Ra}=10^{6}$ as seen from Fig. 5(d). The convection heatlines are denser at the top region of the cavity due to the enhanced buoyancy effect. The area of the passive convection cell is expanded and a small area of the cavity plays an active role on the heat transfer from the hot to the cold wall as seen in the figure. Heat in the large area of the cavity is rotated without affecting on the heat transfer between the hot and the cold surfaces. The increase of Rayleigh number increases the strength of the convection heat transfer and it causes enlargement of the passive convection cell area. This might be the reason behind the fact that the increase of total heat transfer slows down with increasing Rayleigh number.

As it was mentioned before, for a control element in a domain of a convective heat flow, the convective heat rotates due to velocity and/or temperature gradients. The distributions of heat flow vorticity for the cavities with $S_{\mathrm{p}}=0.25$ and different Rayleigh numbers are shown in Fig. 6. The contour plots of heat flow vorticity for $\mathrm{Ra}=10^{2}$ is shown in Fig. 6(a). The values of $\Omega_{t}$ in the regions close to the walls where maximum gradient of velocity exists are higher as compared to the cavity center. The values of $\Omega_{t}$ in the cold wall region are very small since $\theta \cong 0$. The maximum absolute value of heat flow vorticity for the cavity with $\mathrm{Ra}=10^{2}$ and $S_{\mathrm{p}}=0.25$ is found as $\left|\Omega_{t}\right|_{\max }=1.97$. The values of $\Omega_{t}$ increase with increase of Rayleigh number as expected. The maximum absolute value of $\Omega_{t}$ for Ra $=5 \times 10^{3}$ is $\left|\Omega_{t}\right|_{\max }=73.4$. The distribution of $\Omega_{t}$ for the cavity with $\mathrm{Ra}=10^{5}$ becomes completely different. Most of heat from the hot to the cold surface is transferred in the upper region of cavity where the total heatlines are denser and the rest of cavity is passive. A similar distribution can be observed for the cavity with $\mathrm{Ra}=10^{6}$. For the four values of Rayleigh number, the maximum values of heat flow vorticity are observed on the edge of hot wall $(X=0$ and $Y=0.75)$ due to both high velocity and temperature gradients.

Fig. 7, illustrating the distributions of total heatline in the cavities with $\mathrm{Ra}=5 \times 10^{3}$ but the different length of heated plate, indicates the effect of $S_{\mathrm{p}}$ on the passive heat transfer zone. The increase of the heated plate length, located on the left wall, increase the area of heat transfer from the right to the left wall. The passive zone in the cavity prevents the transfer of heat through the shortest path. For the cavity with $S_{\mathrm{p}}>0.25$, heat enters to the cavity flows toward ceiling, then moves parallel to the ceiling and leaves cavity from the right wall due to presence of passive area in where heat rotates. The increase of length of heated plate pushes the passive area to the right wall. It should be reminded that convection heat transfer is dominant in the passive area and then no conduction heat transfer forms in this area. Heat transfer rate through the cavity increases with decrease of the passive area. In order to increase heat transfer from the hot to the cold wall, the area of the passive zone should be reduced.

Many researchers who work on the convective heat transfer use maximum absolute value of streamfunction, $\left|\psi_{\max }\right|$, to evaluate the strength of convection heat transfer. The variation of $\left|\psi_{\max }\right|$ with Rayleigh number for the considered cavities in the present study are drawn and shown in Fig. 8. The increase of Rayleigh number increases the convective heat flow and as a result the absolute values of streamfunction increase. It should be mentioned that the value of the streamfunction depends on the gradients of velocity and mathematically it does not include the gradients of temperature. In natural convection problems, in which heat and fluid flows are coupled, an increase of fluid flow strength increases the convection heat transfer. However, for a forced convection problem, the strength of convection heat transfer may be independent of $\left|\psi_{\max }\right|$. The separation of total heatfunction equation to the conduction and convection heatfunction equations provides a new parameter, $H_{\mathrm{c}}$, for the evaluation of strength of convection heat transfer. The convection heatfunction depends on the variation of $U \theta$ and $V \theta$ rather than the velocity components as $U$ and $V$. Fig. 9 shows the changes of maximum absolute values of the convection heatfunction with Rayleigh number for different values of $S_{\mathrm{p}}$. As seen, the increase of Rayleigh number or $S_{\mathrm{p}}$ enhances convection heat transfer in the cavity. For the present problem, the variation of $\left|H_{\mathrm{cmax}}\right|$ with Rayleigh number and $S_{\mathrm{p}}$ is similar to the changes of $\left|\psi_{\max }\right|$ since the energy and momentum equations are highly coupled. 

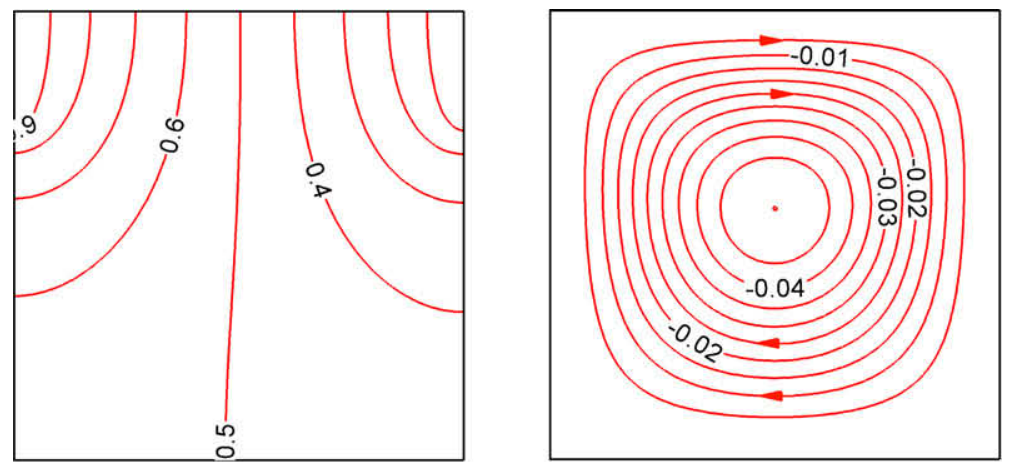

a
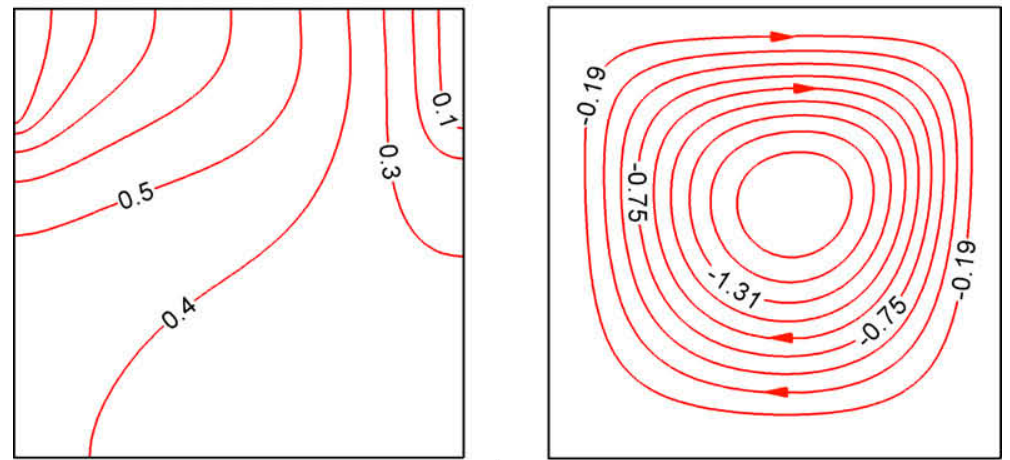

b
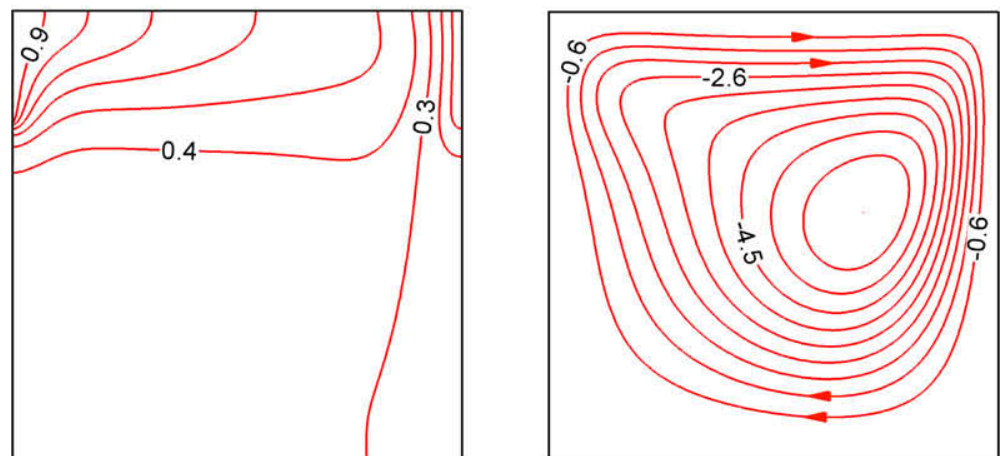

C
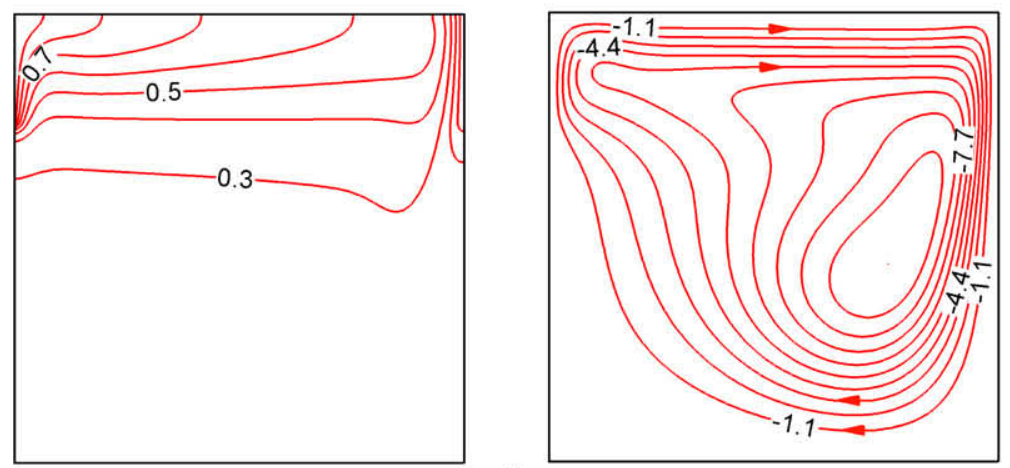

d

Fig. 4. The isotherms (on the left) and streamlines (on the right) for cavity with $S_{\mathrm{p}}=0.25$, (a) $\mathrm{Ra}=10^{2},(\mathrm{~b}) \mathrm{Ra}=5 \times 10^{3},(\mathrm{c}) \mathrm{Ra}=10^{5}$, and (d) $\mathrm{Ra}=10^{6}$.

The variations of $\left|\bar{\Omega}_{t}\right|$ with respect to Rayleigh number and $S_{\mathrm{p}}$ are shown in Fig. 10. The increase of Rayleigh number and heat source length enhances convection heat transfer and consequently the values of $\left|\bar{\Omega}_{t}\right|$ increases. The variation of $\left|\bar{\Omega}_{t}\right|$ with Ra and $S_{\mathrm{p}}$ is very similar to $\left|H_{\mathrm{cmax}}\right|$ with Rayleigh and $S_{\mathrm{p}}$, since the strength of convection heatfunction directly depends on the heat flow vorticity as is seen from Eq. (30).

The variation of average Nusselt number with Rayleigh number for different length of heat source is shown in Fig. 11. The average Nusselt number increases with increase of Rayleigh number. The 

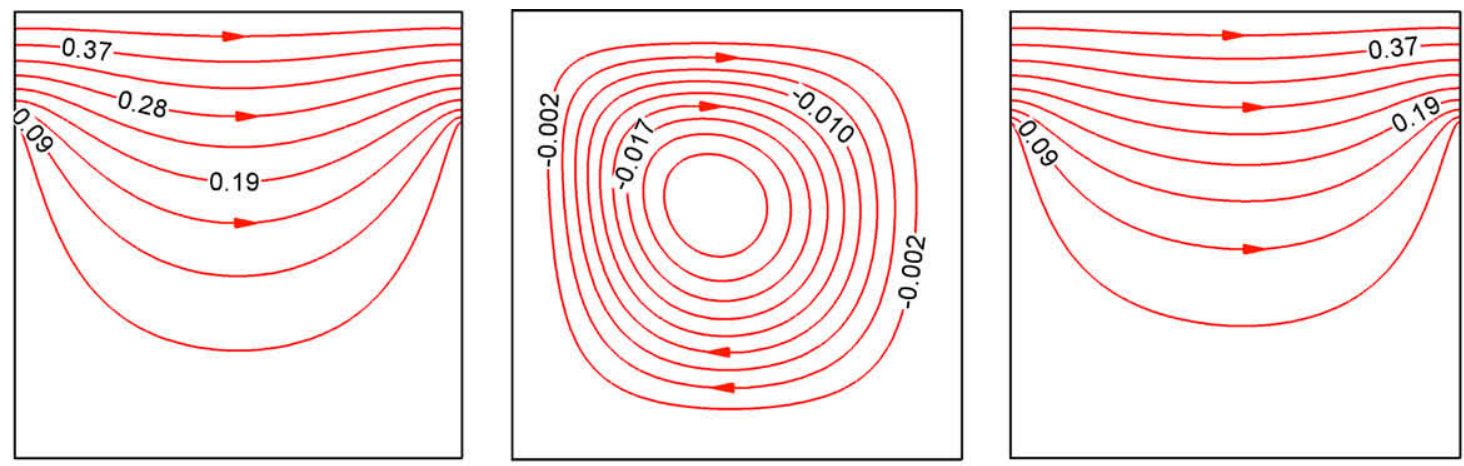

a
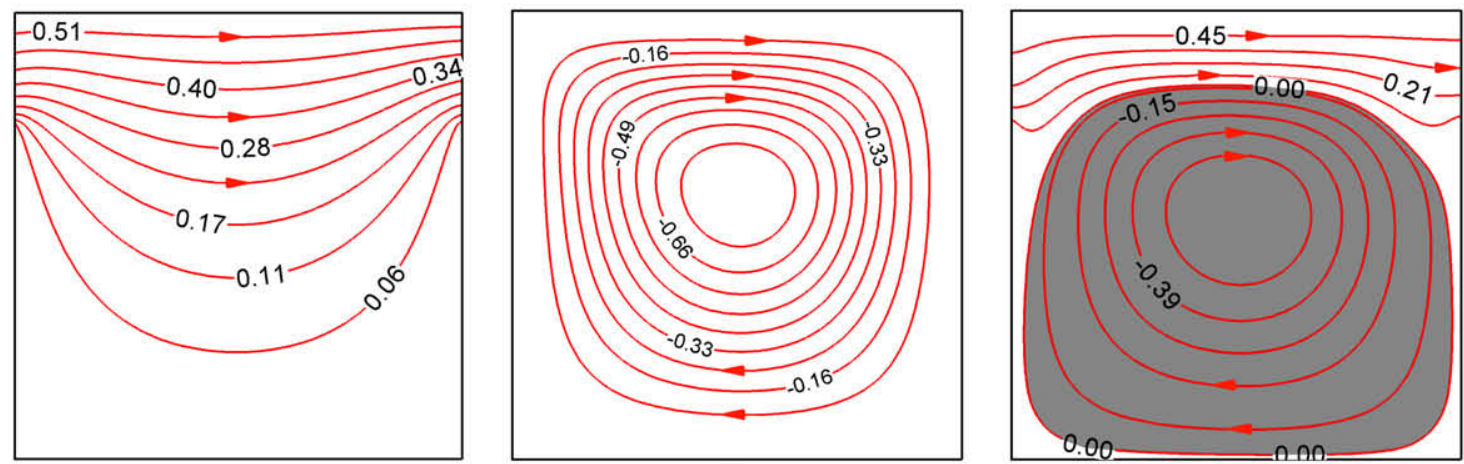

b
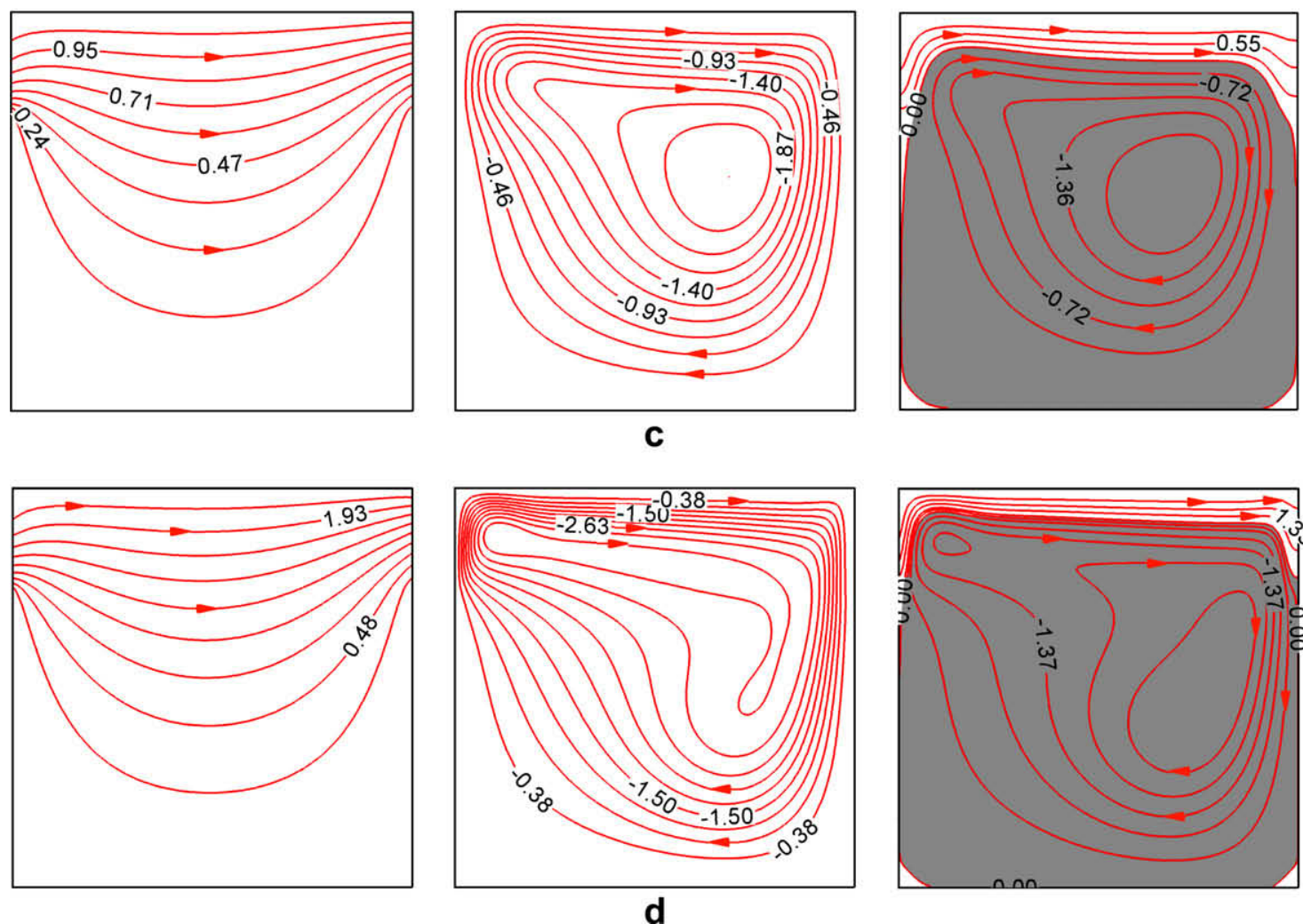

Fig. 5. The diffusion (on the left), convection (on the middle) and total (on the right) heatlines for the cavity with $S_{p}=0.25$, (a) $R a=10^{2}$, (b) b) Ra $=5 \times 10^{3}$, (c) $R a=10^{5}$, and (d) $\mathrm{Ra}=10^{6}$.

increase $S_{\mathrm{p}}$ increases heat flow through the cavity, however; for the cavity with small values of Rayleigh number, the rate of increase is smaller. For $\mathrm{Ra}=10^{2}$ cavity, the increase of $S_{\mathrm{p}}$ from 0.25 to 0.5 causes an increase on the average Nusselt number as $20 \%$, however for $\mathrm{Ra}=10^{6}$ the same increase of $S_{\mathrm{p}}$ improves heat transfer by $55 \%$ due to the enhanced strength of the convection heat transfer. 


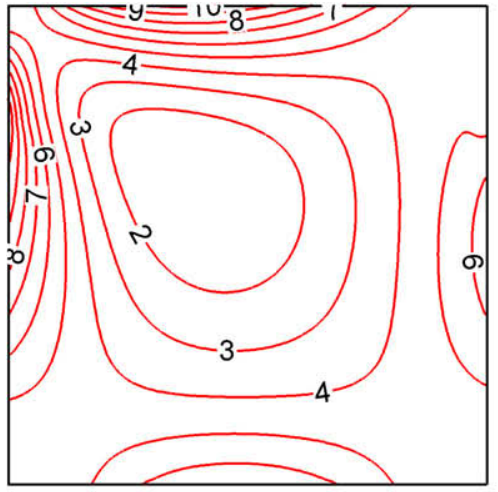

$\mathrm{Ra}=10^{2}$

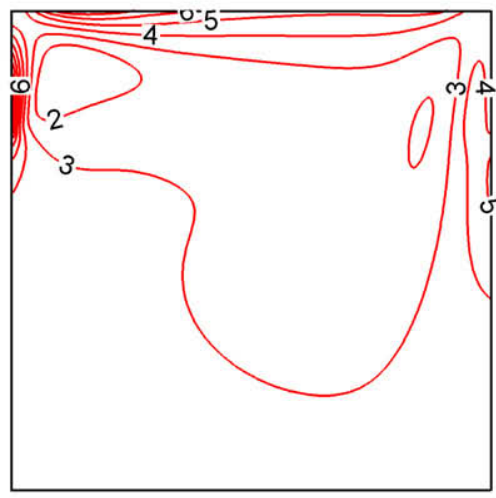

$\mathrm{Ra}=10^{5}$
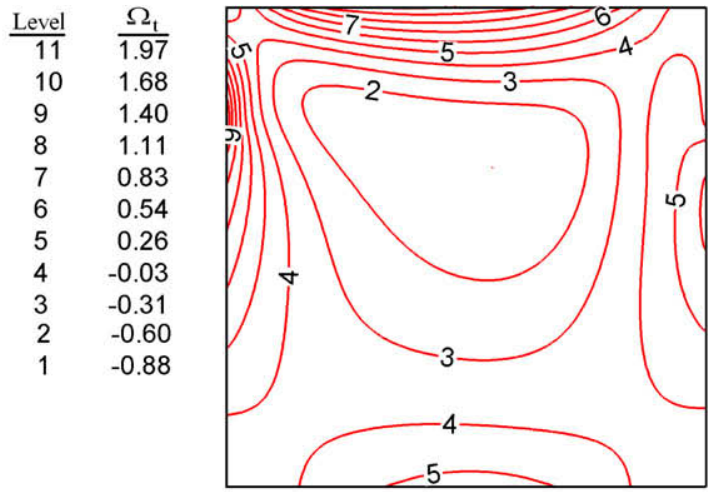

$\mathrm{Ra}=5 \times 10^{3}$
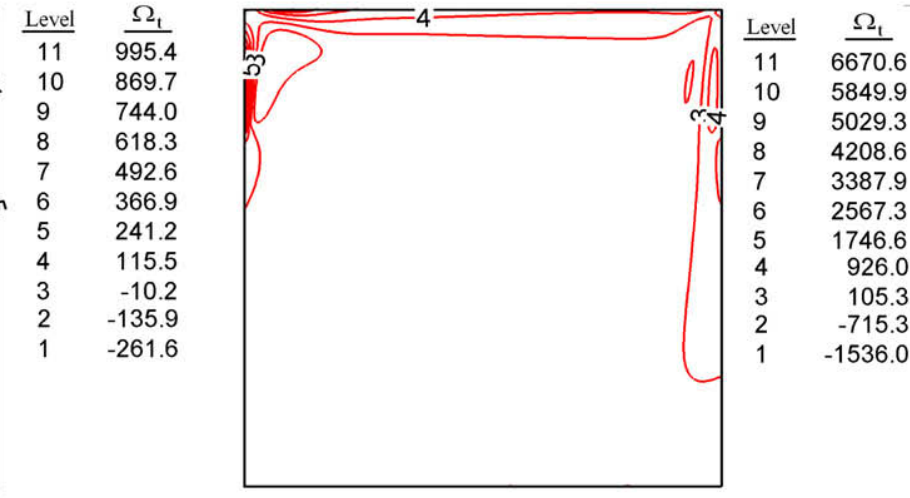

$\mathrm{Ra}=10^{6}$

Fig. 6. The distribution of heat flow rotation for cavity with $S_{p}=0.25$, (a) $R a=10^{2}$, (b) $R a=5 \times 10^{3}$, (c) $R a=10^{5}$, and (d) $R a=10^{6}$.
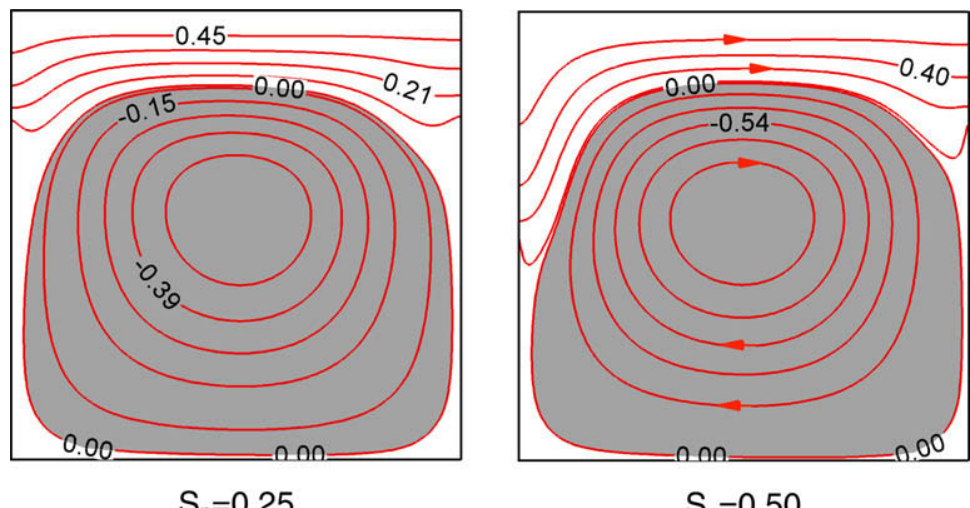

$\mathrm{S}_{\mathrm{p}}=0.50$

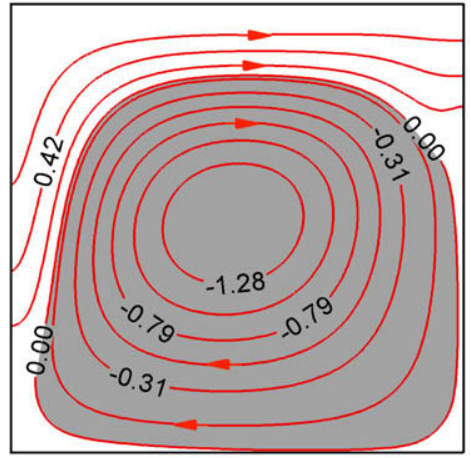

$\mathrm{S}_{\mathrm{p}}=0.75$

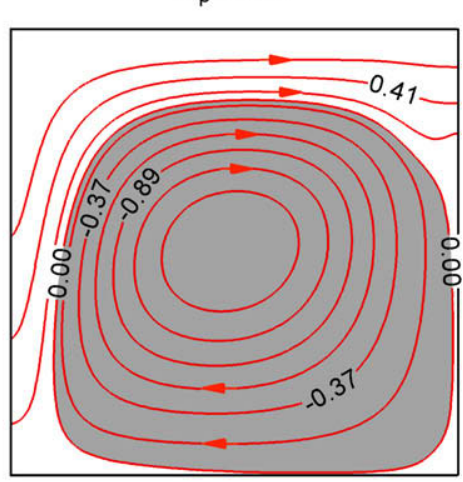

$\mathrm{S}_{\mathrm{p}}=1.0$

Fig. 7. The total heatlines for Ra $=5 \times 10^{3}$ and different values of $S_{\mathrm{p}}$, (a) $S_{\mathrm{p}}=0.25$, (b) $S_{\mathrm{p}}=0.50$, (c) $S_{\mathrm{p}}=0.75$, and (d) $S_{\mathrm{p}}=1.0$. 


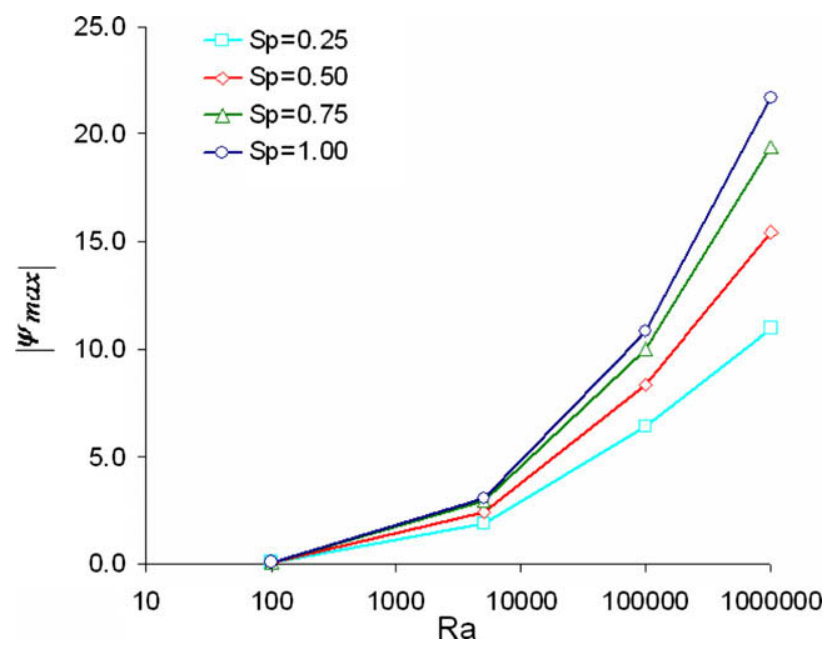

Fig. 8. The variation of $\left|\psi_{\max }\right|$ with Ra for different length of heated surface.

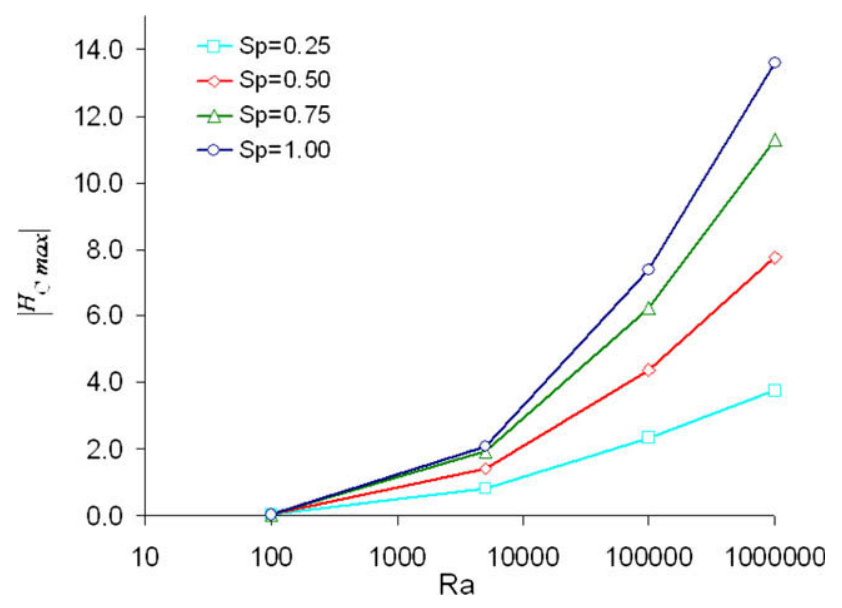

Fig. 9. The variation of $\left|H_{\mathrm{cmax}}\right|$ with Ra for different length of heated surface.

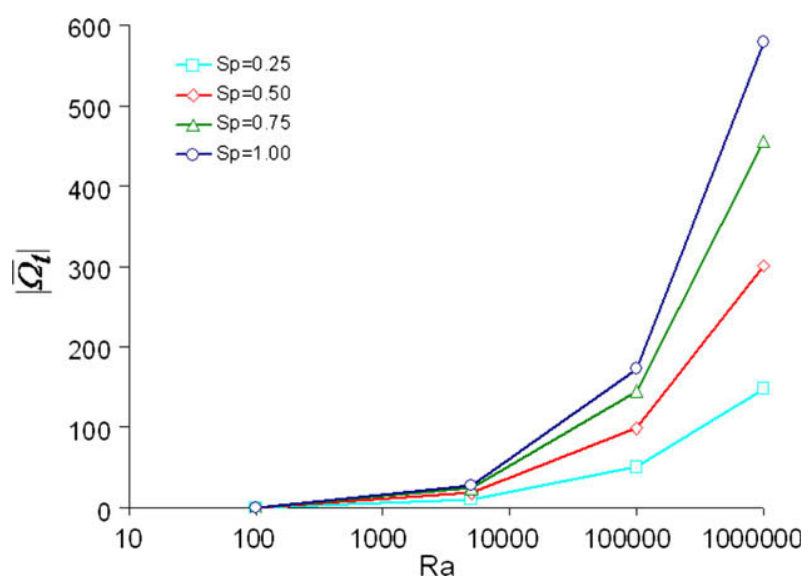

Fig. 10. The variation of $\left|\bar{\Omega}_{t}\right|$ with Ra for different length of heated surface.

The rate of increase of average Nusselt number with $S_{\mathrm{p}}$ slows down for high values of $S_{\mathrm{p}}$. For the cavity with $\mathrm{Ra}=10^{6}$, the rate of increase of the average Nusselt number with $S_{\mathrm{p}}$ from 0.75 to 1.0 is only $6 \%$. For high values of Rayleigh number, the passive portion of the cavity is much bigger so it prevents the direct transfer of heat from the hot to the cold surface.

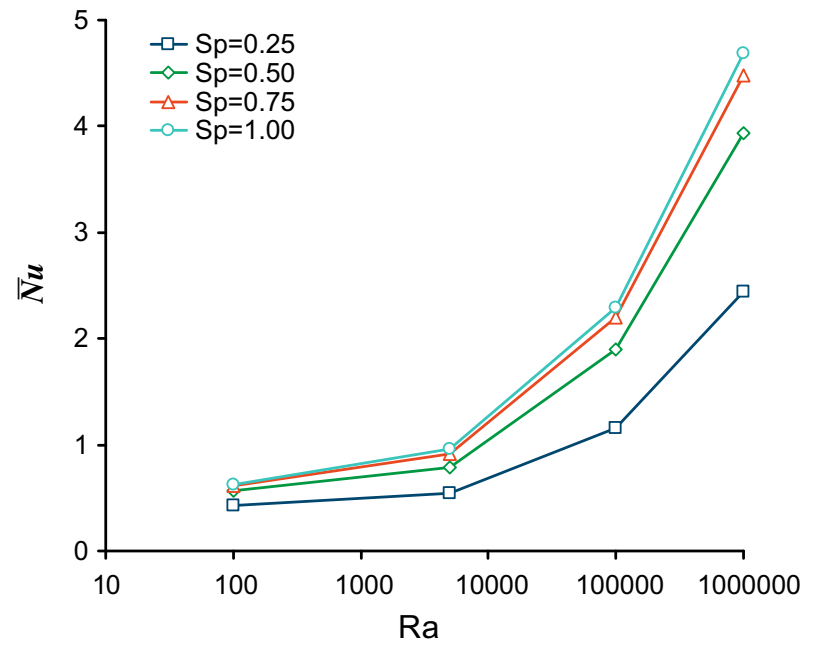

Fig. 11. The changes of mean Nu with Ra for different values of $S_{\mathrm{p}}$.

\section{Conclusions}

The problem of natural convection heat transfer in a square cavity with partially heated vertical walls is numerically solved. The effects of heat source length and Rayleigh number on the heat transfer through the cavity are studied. Literature survey shows that the present study may be the first study handling the separation of total heatlines into diffusion and convection heatlines and presents the definition of the heat flow vorticity. The following remarks can be concluded from the study:

- The superposition rule is successfully applied to separate the total heatfunction equation into the diffusion and the convection heatfunction equations. The paths of conduction and convection modes of heat transfer in the cavity are separately visualized. The diffusion heatfunction equation does not contain any source term and is influenced by the heat flux at the boundaries. The convection heatfunction is influenced by the velocity and temperature fields.

- The comparison of the diffusion, convection and total heatlines for a convective heat flow field reveals the strength of conduction and convection modes in a part or in the entire cavity.

- The sign of the diffusion heatfunction shows the direction of heat transfer while the sign of the convection heatfunction refers to the direction of the heat rotation.

- Similar to the concept of fluid flow vorticity, for a control element in a heat flow domain, the convective heat may have a pointwise rotation. Hence, the concept of heat flow vorticity is proposed in the present study.

- Two regions in the cavity are detected. A region with positive sign of the total heatfunction signifying dominant conduction heat transfer and a convection cell with negative sign of total heatfunction without playing any active role on the heat transfer between the walls. The area of passive cell is enlarged by increase of Rayleigh number.

- The maximum absolute value of the convection heatfunction may be more appropriate than the maximum absolute value of streamfunction for evaluation of convection heat transfer strength.

The present study promotes the similarity between heat and fluid flows. The new defined concepts as convection heatfunction and heat flow vorticity are two important issues on which further studies should be performed. The separation of the diffusion and 
the convection heatfunctions should be applied to other natural and forced convection problems to find out the advantages of heat flow separation better.

\section{References}

[1] S. Kimura, A. Bejan, The heatline visualization of convective heat transfer, ASME J. Heat Transfer 105 (1983) 916-919.

[2] O.V. Trevisan, A. Bejan, Combined heat and mass transfer in a vertical enclosure, ASME J. Heat Transfer 109 (1987) 104-112.

[3] AI.M. Morega, A. Bejan, Heatline visualization of forced convection laminar boundary layers, Int. J. Heat Mass Transfer 36 (1993) 3957-3966.

[4] D.A. Nield, A. Bejan, Convection in Porous Media, second ed., Springer, New York, 1999.

[5] V.A.F. Costa, Bejan's heatlines and masslines for convection visualization and analysis, Appl. Mech. Rev. 59 (2006) 126-145.

[6] V.A.F. Costa, Unification of the streamline, heatline and massline methods for the visualization of two-dimensional transport phenomena, Int. J. Heat Mass Transfer 42 (1999) 27-33.

[7] A. Mukhopadhyay, X. Qin, I.K. Puri, S.K. Aggarwal, Visualization of scalar transport in nonreacting and reacting jets through a unified heatline and massline formulation, Numer. Heat Transfer Part A 44 (2003) 683-704.

[8] F.Y. Zhao, D. Liu, G.F. Tang, Application issues of the streamline, heatline and massline for conjugate heat and mass transfer, Int. J. Heat Mass Transfer 50 (2007) 320-334.

[9] A. Dalal, M.K. Das, Heatline method for the visualization of natural convection in a complicated cavity, Int. J. Heat Mass Transfer 51 (2008) 263-272.

[10] E. Hakyemez, M. Mobedi, H. Oztop, Effects of wall-located heat barrier on conjugate conduction/natural-convection heat transfer and fluid flow in enclosures, Numer. Heat Transfer Part A 54 (2008) 197-220.
[11] M. Mobedi, Conjugate natural convection in a square cavity with finite thickness horizontal walls, Int. Commun. Heat Mass Transfer 35 (2008) 503513.

[12] T. Basak, S. Roy, Role of Bejan's heatlines in heat flow visualization and optimal thermal mixing for differentially heated square enclosures, Int. J. Heat Mass Transfer 51 (2008) 3486-3503.

[13] Q.H. Deng, G.F. Tang, Y. Li, A combined temperature scale for analyzing natural convection in rectangular enclosures with discrete wall heat sources, Int. J. Heat Mass Transfer 45 (2002) 3437-3446.

[14] Q.H. Deng, J. Zhou, C. Mei, Y.M. Shen, Fluid, heat and contaminant transport structures of laminar double diffusive mixed convection in a two-dimensional ventilated enclosure, Int. J. Heat Mass Transfer 47 (2004) 5257-5269.

[15] P. Poulikakos, Natural convection in a confined fluid-filled space driven by a single vertical wall with warm and cold regions, ASME J. Heat Transfer 107 (1985) 867-876.

[16] N. Yücel, H. Türkoglu, Natural convection in rectangular enclosures with partial heating and cooling, Heat Mass Transfer 29 (1994) 471-477.

[17] N. Nithyadevi, N.P. Kandaswamy, J. Lee, Natural convection in a rectangular cavity with partially active side walls, Int. J. Heat Mass Transfer 50 (2007) 4688-4697.

[18] Q.H. Deng, Fluid flow and heat transfer characteristics of natural convection in square cavities due to discrete source-sink pairs, Int. J. Heat Mass Transfer 51 (2008) 5949-5957.

[19] A. Valencia, R.L. Frederick, Heat transfer in square cavities with partially active vertical walls, Int. J. Heat Mass Transfer 32 (1989) 1567-1574.

[20] S. Paolucci, D.R. Chenoweth, Natural convection in shallow enclosures with differentially heated end walls, J. Heat Transfer 110 (1988) 625-634.

[21] C.J. Ho, J.Y. Chang, A study of natural convection heat transfer in a vertical rectangular enclosure with two-dimensional discrete heating: effect of aspect ratio, Int. J. Heat Mass Transfer 37 (1994) 917-925.

[22] G. de Vahl Davis, Natural convection of air in a square cavity; a bench mark numerical solution, Int. J. Numer. Methods Fluids 3 (1983) 249-264. 